\title{
Compartment Syndrome: Diagnosis, Management, and Unique Concerns in the Twenty-First Century
}

\author{
Matthew R. Garner, MD • Samuel A. Taylor, MD • Elizabeth Gausden, MD • John P. Lyden, MD
}

Received: 11 January 2014/Accepted: 19 March 2014 / Published online: 7 June 2014

(C) The Author(s) 2014. This article is published with open access at Springerlink.com

\begin{abstract}
Background: Compartment syndrome is an elevation of intracompartmental pressure to a level that impairs circulation. While the most common etiology is trauma, other less common etiologies such as burns, emboli, and iatrogenic injuries can be equally troublesome and challenging to diagnose. The sequelae of a delayed diagnosis of compartment syndrome may be devastating. All care providers must understand the etiologies, high-risk situation, and the urgency of intervention. Questions/Purposes: This study was conducted to perform a comprehensive review of compartment syndrome discussing etiologies, risk stratification, clinical progression, noninvasive and invasive monitoring, documentation, medical-legal implication, and our step-by-step approach to compartment syndrome prevention, detection, and early intervention. Methods: A literature search was performed using the PubMed Database and the following search terms: "Compartment syndrome AND Extremity," "Compartment syndrome AND Gluteal," and Compartment syndrome AND Paraspinal." A total of 2,068 articles were identified. Filters allowed for the exclusion of studies not printed in English (359) and those focusing on exertional compartment syndrome (84), leaving a total of 1,625 articles available for review. Results: The literature provides details regarding the etiologies, risk stratification, clinical progression, noninvasive and invasive monitoring, documentation, medical-legal implication, and our step-by-step approach to compartment syndrome prevention, detection, and early intervention. The development and progression of compartment syndrome is multifactorial,
\end{abstract}

Electronic supplementary material The online version of this article (doi:10.1007/s11420-014-9386-8) contains supplementary material, which is available to authorized users.

M. R. Garner, MD $(\bowtie) \cdot$ S. A. Taylor, MD • E. Gausden, MD •

J. P. Lyden, MD

Hospital for Special Surgery,

535 East 70th Street,

New York, NY 10021, USA

e-mail: garnerm@hss.edu and as complexity of care increases, the opportunity for the syndrome to be missed is increased. Recent changes in the structure of in-hospital medical care including resident work hour restrictions and the incorporation of midlevel providers have increased the frequency of "signouts" or "patient handoffs" which present opportunities for the syndrome to be mismanaged. Conclusion: The changing dynamics of the health care team have prompted the need for a more explicit algorithm for managing patients at risk for compartment syndrome to ensure appropriate conveyance of information among team members.

Keywords compartment syndrome - intracompartmental pressure $\cdot$ ischemia

\section{Introduction}

Compartment syndrome (CS) occurs when fascial compartment pressures exceed perfusion pressure, leading to irreversible tissue ischemia and necrosis [31]. While literature emphasizes the acute phase, it is important to note that compartment syndrome exists on a spectrum, ranging from acute to chronic. With careful attention to details such as intraoperative positioning, anesthetic choice, and placement of stockings and splints, orthopedic surgeons have the opportunity to modify risk. The sequelae of compartment syndrome have functional, cosmetic, and legal ramifications. Effective treatment begins with early diagnosis.

It is the intent of this article to review common and uncommon causes of compartment syndrome, to highlight the difficulties associated with the identification of patients at risk, and to discuss unique problems surrounding the diagnosis and management of compartment syndrome. Further, we present an algorithm designed to provide a standardized method of patient assessment given the increased frequency of patient "handoffs" that has occurred in the past decade as a consequence of resident work hour restrictions and the increased use of midlevel providers. 


\section{Methods}

A literature search was performed using the PubMed Database and the following search terms: "Compartment syndrome AND Extremity," "Compartment syndrome AND Gluteal," and "Compartment syndrome AND Paraspinal." A total of 2,068 articles were identified. Filters allowed for the exclusion of studies not printed in English (359) and those focusing on exertional compartment syndrome (84), leaving a total of 1,625 articles available for review. Titles and abstracts were then evaluated and articles included based on content and pertinence to the goals of this article. From this group of articles, we also selected reports published over a 10-year period (between 2003 and 2013) reporting compartment syndrome in association with the use of regional anesthesia and having greater than 10 patients.

\section{Results}

\section{Etiology and Risk Factors}

Although most practitioners associate the development of compartment syndrome with an acute injury to the lower leg, it is important to remember that this conditions can develop in many other anatomic regions including the hands, feet, forearms, buttocks, thighs, and even the paraspinal muscles $[1,12,16,31-33,37-39,51]$. A study of 164 patients with acute compartment syndrome found fracture to be the most common primary cause $(69 \%)$, with $30 \%$ of cases associated specifically with tibial shaft fractures [32]. Interestingly, vascular injury, burns, crush injuries, and various iatrogenic causes have been described [5, 17, 31]. Male gender and age less than 35 years have also been shown to be risk factors $[17,32]$; however, this holds true only for traumatic etiologies. Hope and McQueen stratified patients with compartment syndrome into two groups: fracture-related and nonfracture-related (3). The fracture-related group was found to be younger, to have fewer medical comorbidities, and to have a dramatic 13:1 male to female ratio when compared with the atraumatic cohort (3:1). Posterior compartment syndrome of the leg was also noted to be more common in the atraumatic group.

It is important to recognize the factors that contribute to delayed diagnosis of CS such as nonfracture etiologies, mental status, and regional anesthesia. Nonfracture etiologies may lead to delays in diagnoses of up to $13 \mathrm{~h}$ [17]. Regional anesthesia can similarly mask the early signs of compartment syndrome [2, 6, 11, 21, 22, 24]. Obtunded patients may be at higher risk for delayed or missed diagnosis due to their inability to communicate pain, and those with long-acting nerve blocks deserve special attention in the perioperative setting with respect to monitoring compartment pressures.

\section{Diagnosis}

Diagnosis of compartment syndrome has historically been overwhelmingly clinical. Medical educators tend to emphasize the six "P's" of compartment syndrome: pain, pallor, pulselessness, parasthesias, paralysis, and poikothermia. Most clinicians agree that pain out of proportion to injury and increasing analgesic requirements should raise the suspicion of a developing compartment syndrome. Further, parasthesias may occur as an early symptom in acute compartment syndrome, representing a potentially reversible state because peripheral nerves are more sensitive to ischemia than muscle [26]. It is thought that irreversible ischemic changes begin approximately $8 \mathrm{~h}$ after the onset of ischemia [15]. Unfortunately, by the time pallor, pulselessness, and poikothermia are observed, ischemic changes may be irreversible. Delayed diagnosis increases the risk of failed treatment, poor outcomes, additional operations, the possibility of amputation, loss of motion, increased expenses, as well as legal ramifications. Given the subjective nature of these signs and symptoms, we consider the six "P's" as a matter of historic significance and not as a part of any diagnostic algorithm. The diagnosis of compartment syndrome is difficult, but can be based upon both subjective and objective findings. Compartment pressure measurements are useful tools and an important diagnostic adjunct to clinical suspicion, but must not be first line in diagnosing a compartment syndrome in the alert patient with normal sensibility.

There is little difference among models, with regard to the accuracy and reliability of pressure measurements. Most devices use a beveled 18-gauge needle for insertion into muscular the compartment, which is connected to a pressure transducer. Targeting individual compartments, $0.1 \mathrm{ml}$ of saline is flushed through the muscle to create a fluid column. Figure 1 depicts the step-by-step instructions to the general use of this device.

Controversy exists surrounding both the time it takes for irreversible ischemic changes to occur and at what pressure infarction will occur. Sheridan et al. found that, $68 \%$ of 22 patients treated within $12 \mathrm{~h}$ recovered normal lower extremity function compared to only $8 \%$ treated after $12 \mathrm{~h}$ [45]. Finkelstein et al. reported five patients treated after a delay of $35 \mathrm{~h}$ : one patient died of multiorgan failure and the other four required amputation [10]. Despite the evidence that delay in treatment leads to poorer outcomes, it is difficult to determine the exact time of onset for compartment syndrome, making "ischemic time" an educated guess.

With regard to pressure measurements, canine models have shown adequate perfusion despite experimentally controlled compartment pressures of $59 \mathrm{mmHg}$ for $8 \mathrm{~h} \mathrm{[26].}$ Others, however, believe that ischemia occurs at an absolute pressure of 30-50 $\mathrm{mmHg}[14,28-30]$. We advocate the use of pulse pressure, more commonly referred to as " $\Delta \mathrm{P}$," to guide the quantitative diagnosis of compartment syndrome (diastolic blood pressure-intramuscular pressure), with a value below 30 being the cutoff for inadequate perfusion to an extremity [52]. It should be emphasized that this value needs to be considered in conjunction with clinical suspicion. In a prospective study of 97 patients with tibial shaft fractures who all underwent postoperative pressure monitoring of the anterior compartment for $24 \mathrm{~h}$, Janzing and Broos found that using symptoms alone had a specificity of $89 \%$ and sensitivity of $67 \%$, while using $\Delta \mathrm{P}$ under $30 \mathrm{mmHg}$ had 


\section{How to Use Intracompartmental Measurement Devices}

1. Turn monitor on, identify necessary equipment including the pre-filled syringe of sterile normal saline, 18G needle, and tapered chamber stem (image 1).

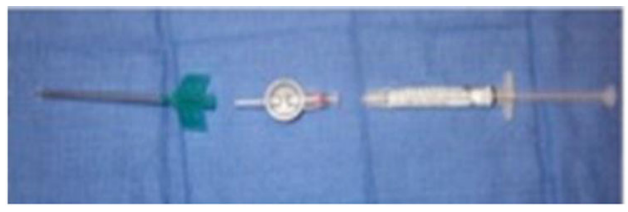

2. Place needle firmly on the tapered chamber stem. Remove cap on pre-filled syringe and secure to the remaining chamber stem.

3. Open the monitor's cover and place the needle, stem and syringe into the unit as shown in Image 2. Close the cover to secure the device.

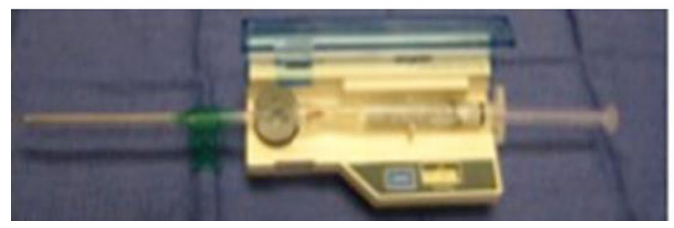

4. Flush fluid from the syringe through the stem and needle until fluid is seen exiting the tip of the needle.

5. Hold the unit at the intended angle against the skin prior to insertion. Press the zero button and wait 2 seconds until the display reads 00.

6. Insert needle into body. Slowly inject less than $3 / 10 \mathrm{cc}$ of saline into the compartment. Wait for the display to reach equilibrium before reading pressure. Then repeat for all compartments.

Fig. 1. Step-by-step instructions to using intracompartmental measurement devices. Note that there may be variability based on a specific device and instructions should be followed.

a specificity of $65 \%$ and a sensitivity of $89 \%$ [19]. Their gold standard was physical exam findings consistent with compartment syndrome at 12 months postoperatively. Using the threshold of $\Delta \mathrm{P}$ under $30 \mathrm{mmHg}, 45.4 \%$ of patients in this study would have undergone fasciotomies, leading to a number of unnecessary operations. Prayson et al. also showed that 8 of 19 asymptomatic tibial shaft fracture patients had $\Delta$ Ps less than $30 \mathrm{mmHg}$ and never developed symptoms or residual deficits [40]. For these reasons, compartment pressure monitoring should be used to confirm clinical suspicion, not as a screening tool for those with an increased risk of developing compartment syndrome.

Continuous intramuscular pressure monitoring with an indwelling catheter is an additional modality that has been advocated in the obtunded or incommunicable patient. McQueen reported a shorter time to fasciotomies when using such monitoring following tibial fractures.

As with any diagnostic procedure, results may be inconsistent, even when measuring pressures in the same patient or specimen. Heckman et al. found significant differences in measurements based on the proximity of the measurement to the fracture site, particularly when pressures were measured within $5 \mathrm{~cm}$ of the fracture site [15]. Based on this data, they recommended measuring as close to the fracture as possible to increase sensitivity of the measurement. Shuler and Dietz have advocated for the use of infrared monitoring of tissue perfusion for the diagnosis of ischemia [47]. This modality is easy and noninvasive; however, it has not yet been validated in the literature.

\section{Spectrum of Compartment Syndrome}

Compartment syndrome exists on a spectrum ranging from acute to late. Acute compartment syndrome should be treated with immediate fasciotomies. Management of late (delayed or missed diagnosis) compartment syndrome is more controversial. The relevance is magnified by the increasing number of patient transfers to tertiary care center for management of both isolated injuries and polytrauma. Reis and Michaelson in 1986 found increased morbidity and mortality in patients that underwent fasciotomies $24 \mathrm{~h}$ or more after compartment syndrome was diagnosed [41]. In these patients presenting more than $24 \mathrm{~h}$ after injury, exposure of 
the necrotic muscle with fasciotomies dramatically increases the risk of infection [10, 42, 43, 45].

In a combat setting, the delay to treatment is a major factor in patient outcomes. In 2008, Ritenour et al. published their results from treating compartment syndromes in wounded soldiers with fasciotomies. The group that had delayed fasciotomies, or fasciotomies that were performed after initial evacuation, had higher rates of muscle excision ( 25 versus $11 \%$ ), amputation (31 versus $15 \%$ ), and mortality (19 versus 5\%) [42]. Similarly, Finkelstein et al. reported a series of five patients who were treated with fasciotomies after delay of at least $35 \mathrm{~h}$, average ischemic time of $56 \mathrm{~h}$. They had one death from multiorgan failure and the other four patients required subsequent amputation [10]. They noted that releasing a compartment in this setting will not reverse ischemic nerve damage or muscle necrosis, but it will convert a previously closed wound to an open one, which exposes necrotic muscle to nosocomial infection.

With increasing frequency, patients are being transferred from facility to facility to allow for advanced treatment at tertiary care centers. Again, this highlights both the importance and the difficulty associated with determining onset of ischemia. Treatment choice must weigh the risk of infection (converting closed wounds to open wounds) with any benefit they would receive from fasciotomies, and when possible, an honest conversation including the risks, benefits, and alternatives of treatment should be had with the patient or those responsible for making medical decisions.

\section{Informed Consent/Patient Expectations}

One of the most overlooked issues surrounding the diagnosis and treatment of compartment syndrome is the patient's right to informed consent. In a recent review of litigation of orthopedic surgeons in the USA, the majority of cases won by the plaintiff cited poor surgeon-patient communication [3]. It is the responsibility of the treating surgeon and team to review with the patient the risks of surgery, treatment options, and the possible long-term sequelae of surgical intervention. A surgeon's urgency can easily lead to poor patient communication and incomplete disclosure. Particular attention should be given to infection and cosmetic deformity. Treatment often involves more than the incident procedure and may require staged debridement and closure or skin grafting, which also impacts length of stay in the hospital. Schmidt was able to show that patients with tibial shaft fractures that developed compartment syndrome had hospital stays on average 6 days longer than those who did not [44]. The surgeon should explain these issues, along with the frequent need for application of negative pressure, or vacuum dressing applications, following fasciotomies so the patient understands the full procedure and the need for repeat operations.

Patient should also be informed of the systemic implications of compartment syndrome. Rhabdomyolysis can negatively impact renal function, and creatinine kinase and serum creatinine levels may need to be followed with serial blood draws. Involvement of larger muscle groups should increase concern for renal sequelae. In 30 soldiers who developed thigh compartment syndrome and underwent fasciotomies, four sustained renal injury and one had renal failure [25].

\section{Anesthetic Considerations}

Regional anesthesia can be of great benefit to orthopedic patients; however, long-acting nerve blocks and epidural anesthesia can be detrimental to patients at risk for developing compartment syndrome. The options for regional anesthesia in orthopedic patients include epidural/spinal anesthesia, peripheral nerve blocks (PNBs), and continuous peripheral nerve blocks (CPNBs). Patient-controlled anesthesia (PCA) infusions also deserve mention given their prevalence in patients undergoing major orthopedic surgery. Communication with the anesthesiologists is paramount to ensure that long-acting blocks and catheters are avoided in high-risk patients. The data regarding the relationship between regional anesthesia and delay in diagnosis of compartment syndrome is limited to case series and case reports (Table 1) $[2,6,8,11,13,22,24,50]$. Mar et al. in 2009 identified 28 case reports and series, 23 of which involved epidural anesthesia, 3 involved patient controlled analgesia, and 2 peripheral nerve blocks [24]. In the 23 studies they reviewed involving epidural anesthesia, they found that 32 of the 35 patients still presented with classic signs and symptoms of compartment syndrome. The authors could not definitively conclude that PNBs delay the diagnosis of compartment syndrome.

Dilute concentrations of local anesthetics in combination with systemic opioid administration can avoid dense motor and sensory blocks $[2,11,18,24]$. Epidural anesthesia delivered and titrated in this manner may avoid masking the intense pain of a developing compartment syndrome. There are a number of case reports of gluteal compartment syndromes as well as lower leg compartment syndromes in the setting of epidural anesthesia that was titrated to the point of motor blockade [18, 20, 21]. Beerle and Rose described a case of prompt diagnosis of compartment syndrome in a patient with an epidural catheter of bupivacaine $0.125 \%$ and fentanyl, titrated for analgesia but preserving motor and sensory function [2]. This case illustrates that regional anesthesia may be used in patients at risk for compartment syndrome, but that dense sensory and motor blocks are ill-advised.

The use of long-acting regional blocks presents another challenge to orthopedic surgeons and anesthetists. Depending on the concentration of anesthetic and the use of preservative, motor and sensory blockade may last from hours to days. The window for diagnosing and treating compartment syndrome is narrow, and when long-acting single block is placed at the time of surgery, the entire progression from early to a late compartment syndrome may be missed clinically. Hyder et al. presented a case of a compartment syndrome in the anterior lower leg following intramedullary nailing of the tibia for a tibial shaft fracture [18]. The patient received a triple nerve block (femoral, obturator and lateral femoral cutaneous) with $0.5 \%$ bupivacaine. Following surgery, the patient was pain-free but reported altered sensation in his foot that the team attributed to the nerve block rather than a developing compartment syndrome. When the paresthesias did not resolve at $48 \mathrm{~h}$, the team measured the compartments and found the anterior compartment to be at $108 \mathrm{mmHg}$. Based on the available literature, it is our 


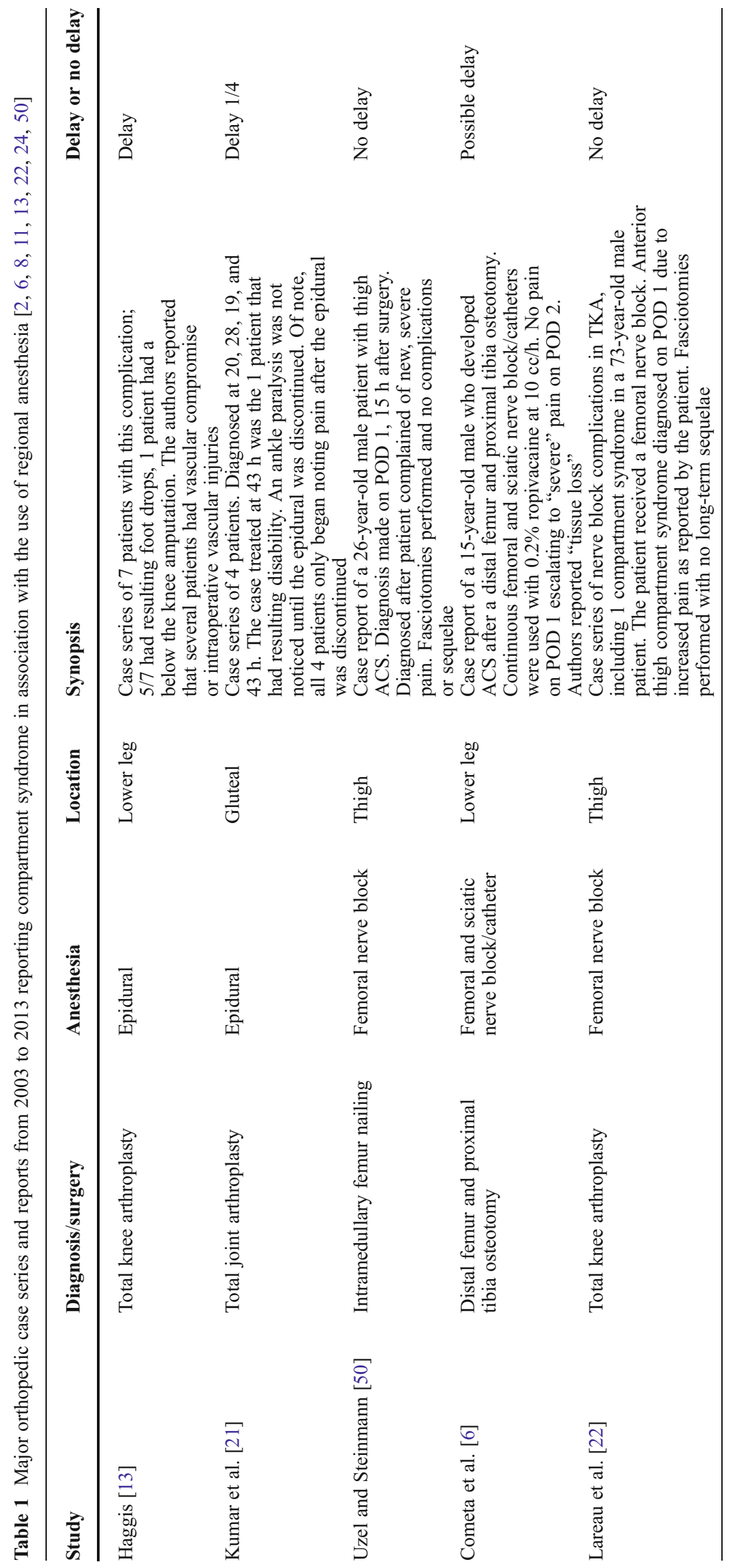




\section{Compartment Check}

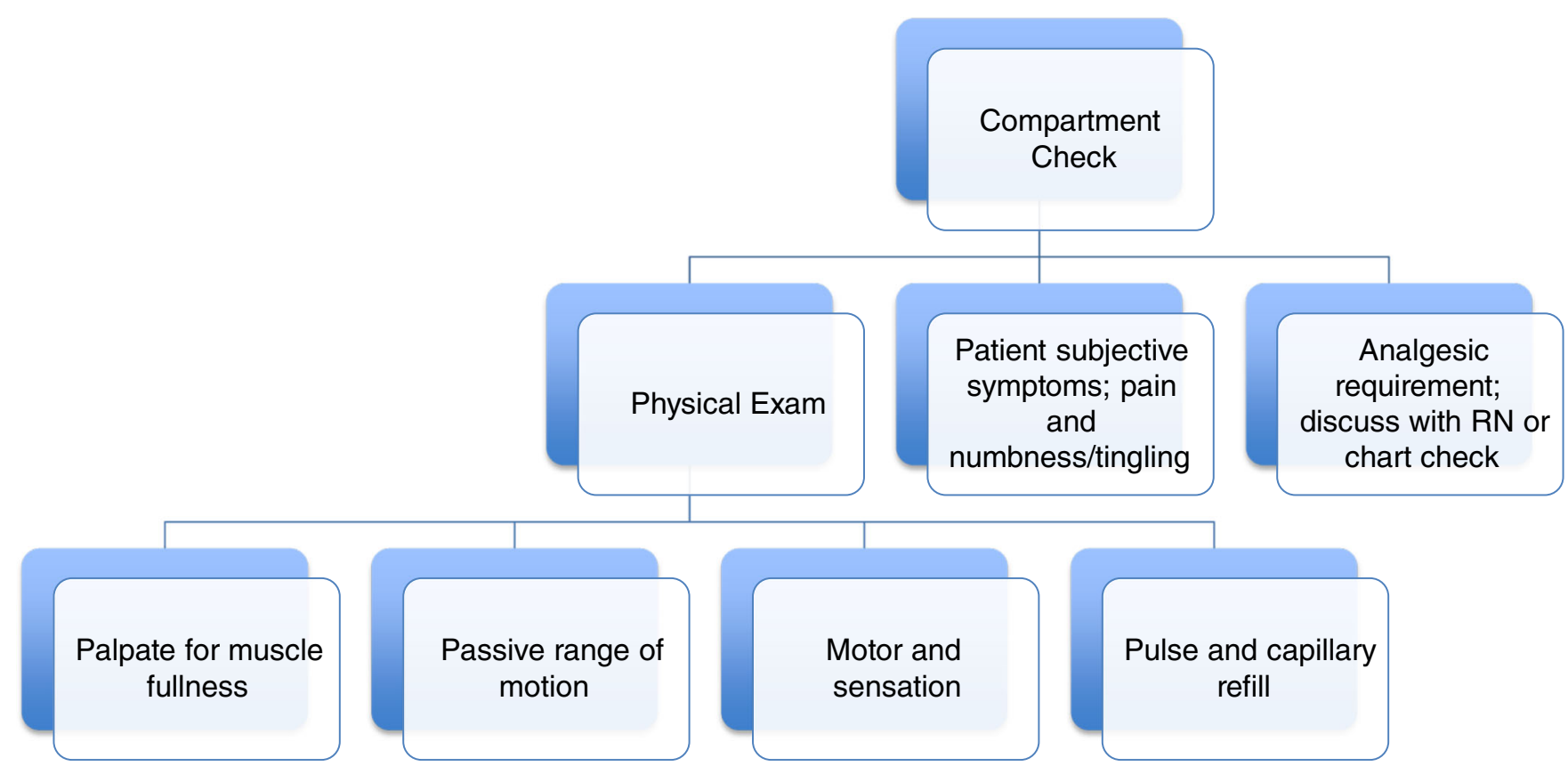

Fig. 2. Basic components of a comprehensive compartment check. The main three tasks are physical exam, evaluating the patient's subjective symptoms, and tracking the patient's analgesic requirements.

opinion that regional anesthesia in patients at risk for developing a compartment syndrome should be limited to shortacting blocks or catheter-delivered analgesics. This will allow for an accurate assessment of pain and neurologic status in the early postoperative period and for dose titration if there is concern for a developing CS.

\section{Documentation and Signout Algorithm}

Resident work hour restrictions and the increased use of midlevel providers have increased the number and frequency of patient handoffs. Patients at risk for compartment syndrome deserve special attention during these signout periods to ensure prompt recognition and treatment when indicated. We advocate the use of the following algorithm when possible:

Step 1: Identify patients at risk and communicate these patients to all members of the team, including nurses, physician assistants, and hospitalists.

Step 2: On-call resident or midlevel provider performs compartment checks every 2 to $4 \mathrm{~h}$ and updates the team's list or handoff documentation with time performed. The compartment check consists of three main components: (1) assessing the patient's pain subjectively; (2) reviewing interim analgesic requirements; and (3) a targeted physical exam (Fig. 2). The targeted physical exam to assess for increased intracompartmental pressure needs to include four crucial components. First, each muscular compartment should be palpated for fullness. We recognize that Shuler et al. recently found that even experienced clinicians are unable to successfully gauge intracompartmental pressures by palpation on physical exam (sensitivity $=54 \%$ ) [46]. This study, however, did not analyze the ability of a practitioner to assess for a change in compartment fullness over time, and we feel strongly that the comparison of examinations at different time points provides essential clinical information. Second, each muscle group should be stretched passively, an exam maneuver that may elicit significant pain in the case of elevated intracompartmental pressure. Then a full neurological evaluation testing motor and sensory function of the affected area is performed. Finally, pulses are palpated and capillary refill is checked to assess for perfusion distal to the site of injury and/or surgery. After the compartment check is complete, the provider should assess the number of criteria that the patient has for diagnosing compartment syndrome. Any one of the four positive physical exam findings described is classified as one criteria: a concerning physical exam. Patient reporting increased pain is a second criterion, and the third is increasing analgesic requirement.

Step 3: Document the compartment check (see below).

Whenever a patient handoff is taking place, we recommend that both care providers examine the patient together 


\section{Intervention Algorithm}

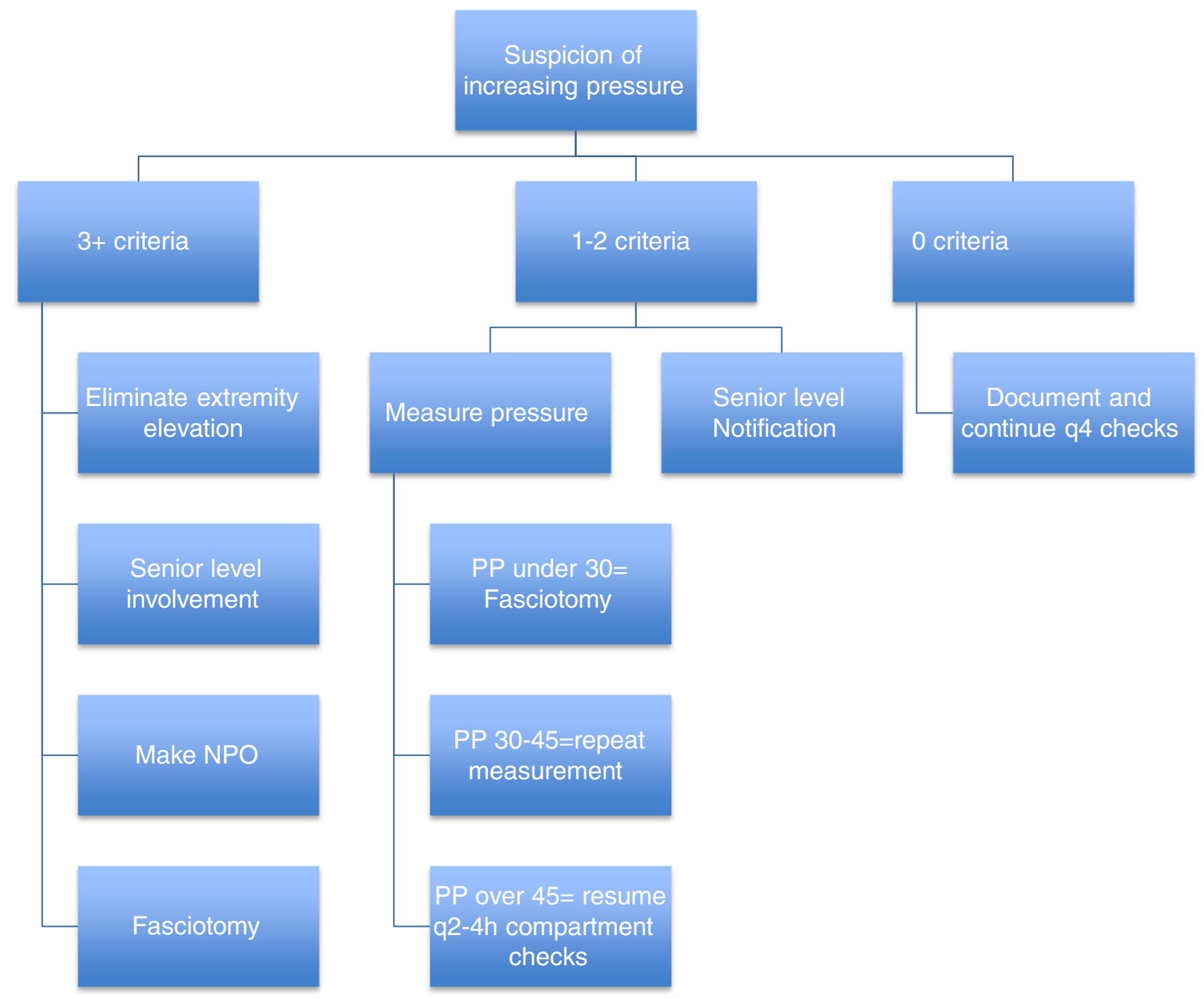

Fig. 3. Our algorithm for interventions based on a patient's subjective report, physical findings, and analgesic requirements.

to establish a baseline exam for the oncoming practitioner, especially if there is clinical concern for a developing compartment syndrome. After reviewing the interim compartment checks and newly assessing the patient, the providers should agree on the number of criteria that the patient has for diagnosing compartment syndrome. In a busy trauma center, this component of the algorithm may prove difficult; however, we believe that it is essential in order to allow for a smooth transition of care and an accurate baseline examination for the oncoming provider. We recognize other limitations of this algorithm, as well. Specifically, our protocol is based on clinical experience and anecdotal evidence and not on scientific study. It does, however, provide a standardized method to approach at-risk patients pre- and postoperatively. Further, this protocol may not be feasible in centers that do not have full-time in-house orthopedic practitioners. In such situations, trained nursing staff may employ a similar algorithm and handoff system. Practitioners at these centers should have a low threshold to return to the hospital for patient assessment if there is any concern of a developing compartment syndrome.

As each compartment syndrome involves assessing and documenting the signs and symptoms of compartment syndrome, the provider should be aware of the appropriate interventions to take depending on the clinical suspicion of compartment syndrome. Figure 3 shows our general algorithm for first-step intervention stratified by clinical suspicion. This is a general guideline to help junior or inexperienced team members act early and attempts to minimize the time delay between onset of symptoms and 
notification of the appropriate provider. Should the examining team member identify a patient with a concerning physical exam, increased reported pain, and/or increased analgesic requirements, they remove any splints or dressings, repeat the examination, and notify a senior team member. From this point, the decision to contact a more senior team member, observe the patient, perform quantitative intracompartmental pressure measurements, or to proceed with surgical intervention can be made based on the hierarchy of the health system.

\section{Documentation and Legal Implications}

Malpractice claims are becoming increasingly relevant to orthopedic surgeons. In 2000, the American Academy of Orthopaedic Surgeons (AAOS) reported that $80 \%$ of orthopedic surgeons had at least one malpractice claim. Despite the prevalence of suits brought against orthopedic surgeons associated with compartment syndrome, there is limited data analyzing the cases and providing advice for preventing future cases. Bhattacharyya recently reviewed claims relating to compartment syndrome of a major insurance company and reported 19 cases involving 16 patients [3]. Six cases were found to be the result of poor physician communication, all of which resulted in an indemnity payment. The authors found that (1) physician documentation of an abnormal finding on neurological exam with no action taken, (2) poor physician communication, (3) increased number of concerning exam findings, and (4) increased time to fasciotomy were statistically significant risk factors for unsuccessful defense in these 19 cases. Based on these findings, the authors concluded that orthopedic surgeons can successfully defend their case if they perform fasciotomies within $8 \mathrm{~h}$ of presentation and act toward treating an evolving compartment syndrome once physical findings are documented.

\section{Special Considerations}

While we have focused a great deal of attention on describing how to monitor patients at high risk for development of compartment syndrome secondary to trauma, several other groups of orthopedic patients deserve mention for their potential to develop compartment syndrome. Although compartment syndrome following total knee and hip arthroplasty is relatively rare, it has been reported in the literature $[16,23$, 38]. Lasanianos et al. reviewed 41 cases of compartment syndrome following arthroplasty, half from total hip arthroplasty and half from total knee arthroplasty [23]. Despite the low incidence in arthroplasty patients, the few patients who develop acute compartment syndromes will be at high risk for delayed diagnosis as they are more likely to be under higher dose epidural anesthesia than trauma patients. Gluteal compartment syndrome is a potential risk following total hip arthroplasty and is attributed frequently to body habitus and prolonged positioning [16]. In a study of 28 patients with gluteal compartment syndrome, half were related to body weight and $21 \%$ were found on the contralateral, "down" side of patients undergoing THA. These findings were particularly prevalent in prolonged revision cases. Gluteal compartment syndrome has also been reported following trauma, iatrogenic vascular injury, pelvic fractures, lateral decubitus or lithotomy positioning in the operating room, overuse or exertion, and epidural analgesic infusion with motor blockade [38]. Sequelae of a missed gluteal compartment syndrome can include sciatic nerve palsy, rhabdomyolysis, and renal failure $[8,16,17]$. Physical examination in the diagnosis of gluteal compartment syndrome may be exceptionally challenging secondary to anatomic considerations. We therefore advocate the liberal use of intracompartmental measurement in these cases.

Compartment syndrome of the foot is most commonly seen following crush injuries, falls from heights, and motor vehicle accidents [36]. Foot compartment syndrome can also complicate up to $10 \%$ of calcaneus fractures [33]. Diagnosis and treatment can be difficult and little consensus exists in the literature. A recent review article by Dodd and Le recommends emergent, three incision fasciotomies at the time of diagnosis [9]. We find that the sequelae of the compartment syndrome including but not limited to contracture, fibrosis, stiffness, and sensory disturbances are often more acceptable to patients than the sequelae of fasciotomies, which may include free tissue transfers [34]. Regardless of the treatment method, we feel strongly that the patient should have a strong understanding of the risks, benefits, and sequelae of both operative and nonoperative management and should be included in the decision making process.

\section{Iatrogenic Considerations}

Orthopedic surgeons also should be aware of the potential to produce compartment syndrome iatrogenically. Tibial nailing is known to be associated with the development of postoperative compartment syndrome. Although intramedullary nailing with manual reduction for tibial shaft fractures causes a transient intraoperative increase in the anterior compartment pressure of the lower leg, two major studies found that the pressures return to baseline following the nail insertion $[35,48]$. Based on these findings, we do not recommend intraoperative compartment measurement, but practitioners should be vigilant with examination and documentation in the early postoperative period.

Another potential iatrogenic etiology of compartment syndrome is the use of tourniquets. Tourniquet syndrome, which represents a reperfusion injury, can occur if tourniquet time exceeds $120 \mathrm{~min}$ and consists of pallor, swelling, and stiffness. Signs and symptoms are typically limited to 1 week and may be prevented by limiting tourniquet time in the operating room or taking 30-min breaks from tourniquet use during long cases to allow for intermittent reperfusion [49].

Malpositioning of patients in the operating room is another unfortunate etiology of iatrogenic compartment syndrome. There have been case reports describing such events from the use of traction tables, specifically injury on the nonoperative side from compression on the support post and elevation leading to hypoperfusion [1,4]. Compartment syndrome from prolonged compression of the down side 
leg in the lateral decubitus position or either leg in the lithotomy position has also been described in the literature $[5,27]$. A complete review of such cases is beyond the scope of this article; however, practitioners need to understand that intraoperative positioning needs to be performed with caution.

Finally, casting, dressings, or ill-fitting compression stockings can also lead to compartment syndrome in orthopedic patients [7]. To minimize the risk at our institution, we use either splints or bivalved casts for all patients presenting with significant swelling or with an injury that has been associated with compartment syndrome. These examples of iatrogenic compartment syndromes are largely preventable and easy to avoid with extra attention to proper positioning, padding, and use of the tourniquet. With heightened awareness, we can decrease the incidence of these cases of compartment syndrome.

\section{Discussion}

In this study, we reviewed the literature regarding prevention, etiology, and diagnosis of compartment syndrome. We have also addressed unique considerations including anesthetic technique, iatrogenic etiologies, and the medical-legal implications of this diagnosis. Despite a wealth of literature surrounding the topic, timely diagnosis of compartment syndrome can be challenging and, in our opinion, is heavily dependent on practitioner experience. Furthermore, work hour restrictions for residents and the incorporation of midlevel providers have increased the number and frequency of "signouts" or "patient handoffs." As such, changes in a physical examination, appropriate conveyance of information among team members, and documentation are particularly important concerns. No studies have addressed how this paradigm shift affects the management and outcomes of these patients and such a study may never exist due to the subjective nature of the diagnosis. We believe that the algorithmic approach to patient evaluation and a standardized escalation policy as presented here may help to limit the impact of these changes in the care of our patients.

Compartment syndrome can be a devastating diagnosis and its development and progression is multifactorial. It is essential that practitioners at all levels must understand the etiologies, high-risk situations, and the immediacy of intervention. As care providers, we have a responsibility to provide early diagnosis and treatment to patients with compartment syndrome and to explain to them the potential sequel of both treatment and management in order to allow for the best possible outcomes and to increase patient satisfaction.

\section{Disclosures}

Conflict of Interest: Matthew R. Garner, MD; Samuel A. Taylor, MD; Elizabeth Gausden, MD; and John P. Lyden, MD, have declared that they have no conflict of interest.
Human/Animal Rights: This article does not contain any studies with human or animal subjects performed by the any of the authors.

\section{Informed Consent: N/A}

Required Author Forms Disclosure forms provided by the authors are available with the online version of this article.

Open Access This article is distributed under the terms of the Creative Commons Attribution License which permits any use, distribution, and reproduction in any medium, provided the original author(s) and the source are credited.

\section{References}

1. Anglen J, Banovetz J. Compartment syndrome in the well leg resulting from fracture-table positioning. Clin Orthop Relat Res. 1994; 301: 239-242.

2. Beerle BJ, Rose RJ. Lower extremity compartment syndrome from prolonged lithotomy position not masked by epidural bupivacaine and fentanyl. Reg Anesth. 1993; 3: 189-190.

3. Bhattacharyya T. The medical-legal aspects of compartment syndrome. J Bone Joint Surg Am. 2004; 4: 864.

4. Carlson DA, Dobozi WR, Rabin S. Peroneal nerve palsy and compartment syndrome in bilateral femoral fractures. Clin Orthop Relat Res. 1995; 320: 115-118.

5. Cascio BM, Buchowski JM, Frassica FJ. Well-limb compartment syndrome after prolonged lateral decubitus positioning. A report of two cases. J Bone Joint Surg Am. 2004; 9: 2038-2040.

6. Cometa MA, Esch AT, Boezaart AP. Did continuous femoral and sciatic nerve block obscure the diagnosis or delay the treatment of acute lower leg compartment syndrome? A case report. Pain Med. 2011; 5: 823-828.

7. Danner R, Partanen K, Partanen J, Kettunen K. Iatrogenic compartment syndrome, A follow-up of four cases caused by elastic bandage. Clin Neurol Neurosurg. 1989; 1: 37-43.

8. David V, Thambiah J, Kagda FH, Kumar VP. Bilateral gluteal compartment syndrome. A case report. J Bone Joint Surg Am. 2005; 11: 2541-2545.

9. Dodd A, Le I. Foot compartment syndrome: Diagnosis and management. J Am Acad Orthop Surg. 2013; 11: 657-664.

10. Finkelstein JA, Hunter GA, Hu RW. Lower limb compartment syndrome: Course after delayed fasciotomy. J Trauma. 1996; 3 : 342-344.

11. Fowler SJ, Symons J, Sabato S, Myles PS. Epidural analgesia compared with peripheral nerve blockade after major knee surgery: A systematic review and meta-analysis of randomized trials. Br J Anaesth. 2008; 2: 154-164.

12. Fulkerson E, Razi A, Tejwani N. Review: Acute compartment syndrome of the foot. Foot Ankle Int. 2003; 2: 180-187.

13. Haggis P. Compartment syndrome following total knee arthroplasty: A report of seven cases. J Bone Joint Surg (Br). 2006; 3: 331 .

14. Halpern AA, Nagel DA. Compartment syndromes of the forearm: Early recognition using tissue pressure measurements. $J$ Hand Surg [Am]. 1979; 3: 258-263.

15. Heckman MM, Whitesides TE Jr, Grewe SR, Rooks MD. Compartment pressure in association with closed tibial fractures. The relationship between tissue pressure, compartment, and the distance from the site of the fracture. J Bone Joint Surg Am. 1994; 9: 1285-1292.

16. Henson JT, Roberts CS, Giannoudis PV. Gluteal compartment syndrome. Acta Orthop Belg. 2009; 2: 147-152.

17. Hope MJ, McQueen MM. Acute compartment syndrome in the absence of fracture. J Orthop Trauma. 2004; 4: 220-224.

18. Hyder N, Kessler S, Jennings AG, De Boer PG. Compartment syndrome in tibial shaft fracture missed because of a local nerve block. J Bone Joint Surg (Br). 1996; 3: 499-500. 
19. Janzing HM, Broos PL. Routine monitoring of compartment pressure in patients with tibial fractures: Beware of overtreatment! Injury. 2001; 5: 415-421.

20. Kontrobarsky Y, Love J. Gluteal compartment syndrome following epidural analgesic infusion with motor blockage. Anaesth Intensive Care. 1997; 6: 696-698.

21. Kumar V, Saeed K, Panagopoulos A, Parker PJ. Gluteal compartment syndrome following joint arthroplasty under epidural anaesthesia: A report of 4 cases. J Orthop Surg (Hong Kong). 2007; 1: 113-117.

22. Lareau JM, Robbins CE, Talmo CT, Mehio AK, Puri L, Bono JV. Complications of femoral nerve blockade in total knee arthroplasty and strategies to reduce patient risk. J Arthroplasty. 2012; 4: 564568.

23. Lasanianos NG, Kanakaris NK, Roberts CS, Giannoudis PV. Compartment syndrome following lower limb arthroplasty: A review. Open Orthop J. 2011; 5: 181-192.

24. Mar GJ, Barrington MJ, McGuirk BR. Acute compartment syndrome of the lower limb and the effect of postoperative analgesia on diagnosis. Br J Anaesth. 2009; 1: 3-11.

25. Masini BD, Racusin AW, Wenke JC, Gerlinger TL, Hsu JR. Acute compartment syndrome of the thigh in combat casualties. $J$ Surg Orthop Adv. 2013; 1: 42-49.

26. Matava MJ, Whitesides TE Jr, Seiler JG 3rd, Hewan-Lowe K, Hutton WC. Determination of the compartment pressure threshold of muscle ischemia in a canine model. J Trauma. 1994; 1: 50-58.

27. Mathews PV, Perry JJ, Murray PC. Compartment syndrome of the well leg as a result of the hemilithotomy position: A report of two cases and review of literature. J Orthop Trauma. 2001; 8: 580-583.

28. Matsen FA 3rd, Mayo KA, Sheridan GW, Krugmire RB Jr. Monitoring of intramuscular pressure. Surgery. 1976; 6: 702-709.

29. Matsen FA 3rd, Wyss CR, Simmons CW. The effects of compression and elevation on the circulation to the skin of the hand as reflected by transcutaneous PO2. Plast Reconstr Surg. 1982; 1: 86-89.

30. McDermott AG, Marble AE, Yabsley RH. Monitoring acute compartment pressures with the S.T.I.C. catheter. Clin Orthop Relat Res. 1984; 190: 192-198.

31. McQueen MM. Acute compartment syndrome. In: Fractures in Adults. 7th ed. Philadelphia: Lippincott Williams \& Wilkins; 2010:689.

32. McQueen MM, Gaston P, Court-Brown CM. Acute compartment syndrome. Who is at risk? J Bone Joint Surg (Br). 2000; 2: 200-203.

33. Myerson M, Manoli A. Compartment syndromes of the foot after calcaneal fractures. Clin Orthop Relat Res. 1993; 290: 142-150.

34. Myerson MS. Management of compartment syndromes of the foot. Clin Orthop Relat Res. 1991; 271: 239-248.

35. Nassif JM, Gorczyca JT, Cole JK, Pugh KJ, Pienkowski D. Effect of acute reamed versus unreamed intramedullary nailing on compartment pressure when treating closed tibial shaft fractures: A randomized prospective study. J Orthop Trauma. 2000; 8: 554558.
36. Ojike NI, Roberts CS, Giannoudis PV. Foot compartment syndrome: A systematic review of the literature. Acta Orthop Belg. 2009; 5: 573-580.

37. Ovre S, Hvaal K, Holm I, Stromsoe K, Nordsletten L, Skjeldal S. Compartment pressure in nailed tibial fractures. A threshold of $30 \mathrm{mmHg}$ for decompression gives 29\% fasciotomies. Arch Orthop Trauma Surg. 1998; 1-2: 29-31.

38. Pacheco RJ, Buckley S, Oxborrow NJ, Weeber AC, Allerton K. Gluteal compartment syndrome after total knee arthroplasty with epidural postoperative analgesia. J Bone Joint Surg (Br). 2001; 5: 739-740.

39. Paryavi E, Jobin CM, Ludwig SC, Zahiri H, Cushman J. Acute exertional lumbar paraspinal compartment syndrome. Spine (Phila Pa 1976). 2010; 25: E1529-33.

40. Prayson MJ, Chen JL, Hampers D, Vogt M, Fenwick J, Meredick $\mathrm{R}$. Baseline compartment pressure measurements in isolated lower extremity fractures without clinical compartment syndrome. $J$ Trauma. 2006; 5: 1037-1040.

41. Reis ND, Michaelson M. Crush injury to the lower limbs. Treatment of the local injury. J Bone Joint Surg Am. 1986; 3: 414-418.

42. Ritenour AE, Dorlac WC, Fang R, et al. Complications after fasciotomy revision and delayed compartment release in combat patients. J Trauma. 2008; 2(Suppl): S153-61. discussion S161-2.

43. Rollins DL, Bernhard VM, Towne JB. Fasciotomy: An appraisal of controversial issues. Arch Surg. 1981; 11: 1474-1481.

44. Schmidt AH. The impact of compartment syndrome on hospital length of stay and charges among adult patients admitted with a fracture of the tibia. J Orthop Trauma. 2011; 6: 355-357.

45. Sheridan GW, Matsen FA 3rd. Fasciotomy in the treatment of the acute compartment syndrome. J Bone Joint Surg Am. 1976; 1: 112-115.

46. Shuler FD. Physicians' ability to manually detect isolated elevations in leg intracompartmental pressure. J Bone Joint Surg Am. 2010; 2: 361.

47. Shuler FD, Dietz MJ. Physicians' ability to manually detect isolated elevations in leg intracompartmental pressure. J Bone Joint Surg Am. 2010; 2: 361-367.

48. Tornetta P 3rd, French BG. Compartment pressures during nonreamed tibial nailing without traction. J Orthop Trauma. 1997; 1: 24-27.

49. Townsend HS, Goodman SB, Schurman DJ, Hackel A, BrockUtne JG. Tourniquet release: Systemic and metabolic effects. Acta Anaesthesiol Scand. 1996; 10: 1234-1237.

50. Uzel AP, Steinmann G. Thigh compartment syndrome after intramedullary femoral nailing: Possible femoral nerve block influence on diagnosis timing. Orthop Traumatol Surg Res. 2009; 4: 309-313.

51. Ward CM. Oedema of the hand after fasciectomy with or without tourniquet. Hand. 1976; 2: 179-185.

52. Whitesides TE, Haney TC, Morimoto K, Harada H. Tissue pressure measurements as a determinant for the need of fasciotomy. Clin Orthop Relat Res. 1975; 113: 43-51. 